\title{
Generalized maximum entropy approach to quasistationary states in long-range systems
}

\author{
Gabriele Martelloni, ${ }^{1, *}$ Gianluca Martelloni, ${ }^{2, \dagger}$ Pierre de Buyl ${ }^{3, \ddagger}$ and Duccio Fanelli ${ }^{2, \S}$ \\ ${ }^{1}$ SISSA, Via Bonomea 286, 34137 Trieste, Italy \\ ${ }^{2}$ Department of Physics and Astronomy, CSDC and INFN University of Florence, via G. Sansone 1, 50019 Sesto Fiorentino, Florence, Italy \\ ${ }^{3}$ Instituut voor Theoretische Fysica, KU Leuven, Belgium \\ (Received 18 July 2015; published 4 February 2016)
}

\begin{abstract}
Systems with long-range interactions display a short-time relaxation towards quasistationary states (QSSs) whose lifetime increases with the system size. In the paradigmatic Hamiltonian mean-field model (HMF) out-of-equilibrium phase transitions are predicted and numerically detected which separate homogeneous (zero magnetization) and inhomogeneous (nonzero magnetization) QSSs. In the former regime, the velocity distribution presents (at least) two large, symmetric bumps, which cannot be self-consistently explained by resorting to the conventional Lynden-Bell maximum entropy approach. We propose a generalized maximum entropy scheme which accounts for the pseudoconservation of additional charges, the even momenta of the single-particle distribution. These latter are set to the asymptotic values, as estimated by direct integration of the underlying Vlasov equation, which formally holds in the thermodynamic limit. Methodologically, we operate in the framework of a generalized Gibbs ensemble, as sometimes defined in statistical quantum mechanics, which contains an infinite number of conserved charges. The agreement between theory and simulations is satisfying, both above and below the out-of-equilibrium transition threshold. A previously unaccessible feature of the QSSs, the multiple bumps in the velocity profile, is resolved by our approach.
\end{abstract}

DOI: 10.1103/PhysRevE.93.022107

\section{INTRODUCTION}

Systems subject to long-range forces are known to display an intriguing feature which manifests both at equilibrium and out of equilibrium [1,2]. As an example starting from out-of-equilibrium initial conditions, long-range systems get trapped in long-lasting quasistationary states (QSSs), whose lifetime diverges with the system size. Importantly, when performing the mean-field limit before the infinite time limit, the system cannot relax towards the Boltzmann-Gibbs equilibrium and stays permanently confined in the QSSs. In this regime, the relevant order parameters take values distinct from those attained at equilibrium, and the system exhibits a large gallery of peculiar anomalies, such as non-Gaussian velocity distributions. QSSs have been reported to occur for a plethora of physical systems for which long-range couplings are at play. These include plasma wave instabilities [3] relevant for fusion devices, self-gravitating systems [4] invoked in the study of nonbaryonic large-scale structure formation in the universe, and free electron lasers [5,6], light sources of paramount importance for their intrinsic flexibility. Elucidating the mechanisms which drive the emergence of QSSs has proved a challenging task that has stimulated a vigorous and still open debate.

QSSs have been interpreted as Vlasov stable stationary states, which evolve because of the collisional, finite $N$ effects [7]. One can hence gain analytical insight into the QSSs by resorting to a Vlasov based maximum entropy scheme, as originally pioneered by Lynden-Bell and Wood in their seminal paper [8]. The aforementioned method has

\footnotetext{
*gmartell@sissa.it

${ }^{\dagger}$ gianluca.martelloni@unifi.it

†pierre.debuyl@chem.kuleuven.be

§uccio.fanelli@unifi.it
}

been successfully applied both to paradigmatic toy models [9] and real world systems [5], allowing us to unravel a rich zoology of out-of-equilibrium attributes that encompass phase transitions [10] and phase reentrances [11]. Remarkably, not a single adjustable parameter is employed in the theory, which has therefore a fully predictive value. Despite the general agreement with the results of direct simulations, deviations can be detected when the ergodic hypothesis, which ultimately underlies the maximization procedure, fails. Dynamical effects need to be properly included in the theory to eventually improve its inherent prognostic ability. Working along these lines, the so-called core-halo solution was proposed in Ref. [12]. The mechanisms of core-halo formation have similarities to the process of evaporative cooling: macroscopic density waves propagate in the hosting medium. Particles matching the resonant conditions can gain energy at the detriment of collective modes and thus leave the inner core to populate a diffusing halo. On the other hand, the loss of energy drives a condensation into a low energy state of the particles constituting the bulk. Due to the Vlasov constraints, the core cannot freeze by eventually collapsing into the minimum of the potential. It in turn approaches the maximum allowed phase space density, as imposed by the assigned initial conditions. The method results in a semianalytical strategy to accurately characterize the QSS state in the regime where particles bunch inside the separatrix of a collective resonance (magnetized phase, as described below). An alternative strategy inspired by the Bernstein-Greene-Kruskal theory of plasma physics [13] was proposed in Ref. [14] to characterize the QSSs. The approach yields a dynamical description of the self-consistent steady states. The sought stationary regimes are designed to obey the initial energy distribution. It should be, however, emphasized that this is an approximate technique, which does not account for the relaxation driven by the actual Vlasov (or $N$-body) dynamics. Notwithstanding the undoubted success of the theories mentioned above, we are, however, far from having 
said the final word on the genesis of QSSs. With reference to the celebrated Hamiltonian mean-field (HMF) model [15], the classically recognized arena for challenging QSS studies, pronounced bumps have been, for instance, reported to spontaneously set in for the homogeneous QSS regime [16,17]. These vortices, or phase space clumps, imply that the relaxation is incomplete. In Ref. [18], a nonlinear superposition of counterpropagating Lynden-Bell solutions was employed to describe the complex clustering observed in numerical simulations. This is an interesting ad hoc recipe, which, however, lacks solid first principles grounds. Starting from these premises, the aim of this paper is to discuss an extension of the original Lynden-Bell approach to reconcile theory and empirical observations. Although we will develop the analysis for the HMF model, the method is general and could be, in principle, mutuated to those settings, from plasma physics to free electron lasers, where the QSSs have been shown to occur.

\section{THE HAMILTONIAN MEAN-FIELD MODEL AND LYNDEN-BELL'S THEORY}

Let us start by introducing the HMF model, which describes the coupled dynamics of $N$ classical rotators. The Hamiltonian reads

$$
H=\frac{1}{2} \sum_{j=1}^{N} p_{j}^{2}+\frac{1}{2 N} \sum_{i, j=1}^{N}\left[1-\cos \left(\theta_{j}-\theta_{i}\right)\right],
$$

where $\theta_{j}$ stands for the orientation of the $j$ th rotor and $p_{j}$ is its conjugate momentum. To monitor the evolution of the system, one can rely on the magnetization, a global order parameter defined as $M=|\mathbf{M}|=\left|\sum \mathbf{m}_{i}\right| / N$, where $\mathbf{m}\left(\theta_{i}\right)=$ $\left(\cos \theta_{i}, \sin \theta_{i}\right)$ represents the local magnetization vector. As already recalled, starting from out-of-equilibrium initial conditions, the system gets frozen in a QSS, whose time duration diverges with the number of simulated particles $N$. When the limit $N \rightarrow \infty$ is performed before the infinite time limit $(t \rightarrow \infty)$, the system is stuck in the QSS and cannot proceed towards its associated equilibrium configuration [19]. In other words, QSSs can be interpreted as stationary stable equilibria of the system in its mean-field continuum representation. This observation immediately translates into a rigorous route to analytically inspect the peculiar out-of-equilibrium dynamics of systems subject to long-range couplings.

To this end, we preliminary recall that, in the $N \rightarrow \infty$ limit, the $N$-particle dynamics of Hamiltonian (1) (similarly for a Hamiltonian of the same class) yields the following Vlasov equation:

$$
\frac{\partial f}{\partial t}+p \frac{\partial f}{\partial \theta}-\frac{d V}{d \theta} \frac{\partial f}{\partial p}=0
$$

where $f(\theta, p, t)$ denotes the microscopic one-particle distribution function and

$$
\begin{gathered}
V(\theta)[f]=1-M_{x}[f] \cos (\theta)-M_{y}[f] \sin (\theta), \\
M_{x}[f]=\int_{-\pi}^{\pi} \int_{-\infty}^{\infty} f(\theta, p, t) \cos \theta d \theta d p, \\
M_{y}[f]=\int_{-\pi}^{\pi} \int_{\infty}^{\infty} f(\theta, p, t) \sin \theta d \theta d p .
\end{gathered}
$$

The specific energy $h[f]=\iint\left(p^{2} / 2\right) f(\theta, p, t) d \theta d p-$ $\left(M_{x}^{2}+M_{y}^{2}-1\right) / 2$ and momentum $P[f]=\iint p f(\theta, p, t)$ $d \theta d p$ functionals are conserved quantities, together with mass normalization.

The main idea of the Lynden-Bell maximum entropy theory is to coarse grain the microscopic one-particle distribution function $f(\theta, p, t)$. It is then possible to associate an entropy to $\bar{f}$, the coarse-grained version of the single-particle distribution. The sought statistical equilibrium can be determined by maximizing such an entropy while imposing the conservation of the Vlasov dynamical invariants $[8,20,21]$.

We shall here assume that the initial distribution is of the water-bag type. We will in particular restrict the analysis to a rectangular water bag in the $(\theta, p)$ plane, centered in the origin and of respective half widths $\Delta_{\theta}$ and $\Delta_{p}$. The distribution $f$ takes therefore just two values: $f_{0}=1 /\left(4 \Delta_{\theta} \Delta_{p}\right)$ inside the boundaries of the rectangle and zero outside. The water-bag geometry is entirely specified once the energy $h[f]=e$, and the initial magnetizations $\mathbf{M}_{0}=\left(M_{x 0}, M_{y 0}\right)$ are assigned.

The Vlasov time evolution can reshape the boundaries of the water bag while preserving the area inside it. The distribution stays therefore at two levels $\left(0, f_{0}\right)$ as time progresses. In this two-level representation, the mixing entropy per particle associated with $\bar{f}$ reads

$s[\bar{f}]=-\iint \frac{\bar{f}}{f_{0}} \ln \frac{\bar{f}}{f_{0}}+\left(1-\frac{\bar{f}}{f_{0}}\right) \ln \left(1-\frac{\bar{f}}{f_{0}}\right) d \theta d p$,

which follows from a straightforward combinatorial analysis $[8,20]$. The Lynden-Bell recipe amounts to solving the following constrained variational problem:

$S(e, \sigma)=\max _{\bar{f}}\left(s[\bar{f}] \mid h[\bar{f}]=e ; P[\bar{f}]=\sigma ; \iint d \theta d p \bar{f}=1\right)$.

To carry out the calculations one needs to introduce three Lagrange multipliers, $\beta / f_{0}, \lambda / f_{0}$, and $\mu / f_{0}$, for, respectively, energy, momentum, and normalization. This leads to the following analytical form of the distribution:

$$
\bar{f}(\theta, p)=f_{0} \frac{e^{-\beta\left\{p^{2} / 2-\mathbf{M}[\bar{f}] \cdot \mathbf{m}(\theta)\right\}-\lambda p-\mu}}{1+e^{-\beta\left\{p^{2} / 2-\mathbf{M}[\bar{f}] \cdot \mathbf{m}(\theta)\right\}-\lambda p-\mu}},
$$

which differs from the Boltzmann-Gibbs expression because of the "fermionic" denominator that originates from the specific nature of the entropy (6). By inserting expression (8) into energy, momentum, and normalization constraints and by making use of the definition of magnetization, one can obtain a set of implicit equations in the unknowns $\beta, \lambda, \mu, M_{x}$, and $M_{y}$. Such a system can be solved, for any supplied values of the energy $e$ and the initial magnetization $M_{0}$, to determine the Lagrange multipliers, together with $M_{x}$ and $M_{y}$, for which the Lynden-Bell entropy is extremal.

Interestingly, the calculation sketched above enables one to detect an out-of-equilibrium phase transition [10] in the reference plane $\left(M_{0}, e\right)$ : when assigning the initial magnetization and decreasing the energy density $e$, the system experiences a switch from a homogeneous (zero magnetization) to a nonhomogeneous (magnetized) QSS. Consequently, the plane of parameters $\left(M_{0}, e\right)$ can be partitioned into two adjacent 
zones respectively associated with ordered and disordered phases. The transition line, which separates the aforementioned regimes, is a collection of critical points, as determined by the Lynden-Bell theory. Both first- and second-order phase transition are identified, which merge together into a tricritical point. The details of the transition have been more recently revisited through the core-halo approach [12], resulting in a refined description of the scrutinized phenomenon. Above the threshold, when the system is predicted to relax towards a homogeneous QSS, two symmetric bumps appear in the velocity distribution, as recorded via $N$-body and Vlasov based simulations. This is the imprint of a collective effect which yields the formation of two clusters in the $(\theta, p)$ plane. Neither the Lynden-Bell theory nor the core-halo semianalytic approach could provide a plausible account for the spontaneous rise of the bumps, an observation which remains to date unexplained. The aim of this paper is to discuss an extension of the Lynden-Bell maximum entropy scheme, which accommodates for a set of additional Vlasov constraints that prove necessary to benchmark theory and simulations. We will here exclusively report numerical simulations of the Vlasov equation, the continuum counterpart of the original $N$-body Hamiltonian (1). All simulations were performed with the VMF90 program [22]. Provided $N$ is sufficiently large, discrete $N$-body simulations and their corresponding Vlasov homologue are virtually identical [16]. Incidentally, we will also show that the velocity profiles recorded above the threshold return a rather intricate gallery of possible structures, ranging from two to multiple bumps, an observation which contributes to significantly enriching the phenomenology so far reported in the literature [23].

\section{EXTENDED LYNDEN-BELL THEORY}

Consider the $n$th moment $\left\langle\eta(\theta, p ; t)^{n}\right\rangle$ of the distribution function defined as

$$
\eta_{n}(t) \equiv\left\langle\eta(\theta, p ; t)^{n}\right\rangle=\int_{-\pi}^{\pi} \int_{-\infty}^{\infty} \eta(\theta, p ; t)^{n} f(\theta, p) d \theta d p,
$$

where

$$
\eta(\theta, p ; t) \equiv \frac{p^{2}}{2}-\mathbf{M}(t) \cdot \mathbf{m}(\theta)
$$

is the one-particle Hamiltonian that depends on the instantaneous value of the magnetization $\mathbf{M}(t)$, yielding the appropriate equations of motion for particles. Starting from out-of-equilibrium initial conditions of the water-bag type, with specified energy and initial magnetization, one can follow the time course of $\left\langle\eta(\theta, p ; t)^{n}\right\rangle$ for arbitrary choices of the integer $n$. The typical result of a large campaign of simulations is depicted in Fig. 1: after a violent relaxation, which is customarily termed the sudden initial evolution, the moments approach stationary plateaus characterized by well-defined average values. In formulas, $\lim _{t \rightarrow \infty} \eta_{n}(t)=\epsilon_{n}$, where $\epsilon_{n}$ are scalars determined by the simulations. The Vlasov dynamics is sampling its equilibrium solution: the modest oscillations displayed by $\eta_{n}(t)$ around their mean values $\epsilon_{n}$, as shown in Fig. 1, reflect the local wandering of the trajectory inside
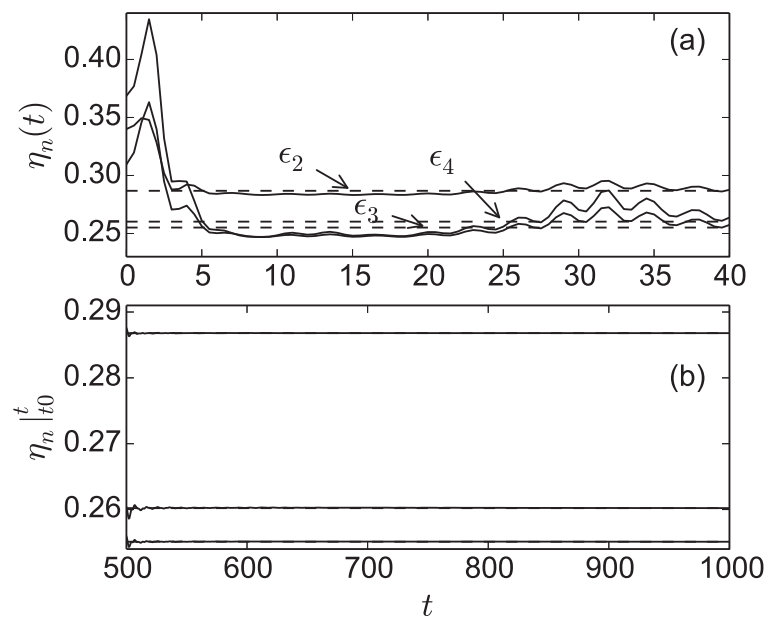

FIG. 1. (a) Time evolution of the first few moments $\eta_{n}$ with $n=2,3,4$. After a violent relaxation the moments converges to their asymptotic values $\epsilon_{n}$, shown by dashed lines. The results refer to a choice of the parameter which yields homogeneous QSSs. Similar observation holds for the magnetized QSS phase. Notice that in the homogeneous QSS $\left\langle\eta(\theta, p ; t)^{n}\right\rangle \equiv\left\langle p^{2 n}\right\rangle / 2^{n}$. Imposing the conservation of $\eta_{n}$ is equivalent to assuming the (rescaled) even velocity moments are invariant. (b) Running averages $\left.\eta_{n}\right|_{t_{0}} ^{t}=$ $\left(t-t_{0}\right)^{-1} \int_{t_{0}}^{t} \eta_{n}\left(t^{\prime}\right) d t^{\prime}$ in the time interval $(250,1000)\left(\right.$ i.e., $\left.t_{0}=250\right)$.

the target basin of attraction. In the discrete realm, finite $N$ effects are indeed responsible for the subsequent slow evolution towards the asymptotic Boltzmann equilibrium.

Working in the continuum setting implies silencing endogenous finite $N$ corrections and consequently preventing the system from leaving the collisionless Vlasov equilibria. These are the QSSs that we aim at characterizing and which should be self-consistently coerced to match the average values $\epsilon_{n}$ for the asymptotic moments $\eta_{n}$, as measured from Vlasov simulations. Building on this observation, we elaborate on a possible modification of the Lynden-Bell maximum entropy solution, which is constrained to reproduce the moments of the distribution, treated as additional pseudoconserved quantities. The moments themselves are not Vlasov invariant, but they contribute as dynamical constraints to effectively delimit the portion of available phase space. In the following, for purely demonstrative purposes, we will make use of the average values for $\eta_{n}(t)$, as resulting from the simulations. In general it is, in principle, possible to construct a dedicated moment closure scheme which enables one to estimate the sought quantities, yielding a fully predictive approach to the QSS characterization [24]. This task is not explicitly carried out in this paper, and consequently, the proposed theory has currently semianalytic value. As an additional remark, we emphasize again that we have not altered the intimate formulation of Lynden-Bell's entropy and thus still rely on the idea that it represents the appropriate quantity to describe the process of Vlasov relaxation. We have instead enlarged the set of constraints that enter the whole optimization procedure. While we do not provide at present a closed characterization for $\epsilon_{n}$, they could be, in principle, self-consistently assessed. 
Mathematically, we shall study the generalized variational problem

$$
\begin{aligned}
& S\left(e, \sigma, \epsilon_{2 n}\right)=\max _{\bar{f}}(s[\bar{f}] \mid h[\bar{f}]=e ; P[\bar{f}]=\sigma ; \\
& \left.\iint d \theta d p \bar{f}=1 ;\left\langle\eta(\theta, p)^{n}\right\rangle=\epsilon_{n} \forall n \geqslant 2\right) .
\end{aligned}
$$

Here, the dependency on time of $\eta(\theta, p ; t)$ has been removed, as the theory only considers asymptotically stationary regimes; hence $\eta(\theta, p)=\lim _{t \rightarrow \infty} \eta(\theta, p ; t)$. Formally, we hence replace $\mathbf{M}$ in the definition of $\eta$ with $\mathbf{M}_{Q S S}$, the value self-consistently determined via the maximum entropy procedure. Several comments are mandatory at this points. First, notice that $\eta$ reduces to $p^{2} / 2$ in the region of parameters that takes the systems towards a homogenous QSS. In this case, the constraints are linear in the unknown distribution function $\bar{f}$, and the variational calculation is both exact and straightforward. Conversely, in the region where the QSS is magnetized, one should, in principle, account for the nonlinearities stemming from the implicit dependence of $\mathbf{M}$ on $\bar{f}$, in the definition of $\eta$. To proceed in the analysis, we choose to consider $\mathbf{M}$ fixed in the expression of $\eta$ and assume it to coincide with the self-consistently determined value $\mathbf{M}_{Q S S}$. In doing so, we formally neglect the functional variations $\frac{\delta M}{\delta f}$ when carrying out the maximization scheme. Intuitively, the additional constraints can be imagined to influence locally the sought optimum, while the magnetization appears to be primarily determined by the truly conserved quantities. The validity of this operating ansatz can be $a$ posteriori assessed via comparison with numerical simulations.

By introducing the Lagrange multipliers $v_{n} / f_{0}$ to account for the additional imposed constraints and performing the calculation, one ends up with the following expression for the extremal distribution function $f_{Q S S}(\theta, p) \equiv \bar{f}(\theta, p)$ :

$$
f_{Q S S}=f_{0} \frac{e^{-\beta\left[p^{2} / 2-\mathbf{M}_{Q S S} \cdot \mathbf{m}(\theta)\right]-\sum_{n} v_{n} \eta(\theta, p)^{n}-\mu}}{1+e^{-\beta\left[p^{2} / 2-\mathbf{M}_{Q S S} \cdot \mathbf{m}(\theta)\right]-\sum_{n} v_{n} \eta(\theta, p)^{n}-\mu}} .
$$

Notice that we have set $\lambda=0$, as it immediately follows for symmetry reasons. The Lagrange multipliers, together with the unknown magnetization amount, can be determined by imposing the selected constraints [25]. These are the conserved quantities, energy and normalization, and the stationary values for the even moments of the distribution:

$$
\begin{aligned}
\int_{-\pi}^{\pi} \int_{-\infty}^{\infty} f_{Q S S} d \theta d p & =1, \\
\int_{-\pi}^{\pi} \int_{-\infty}^{\infty} \frac{p^{2}}{2} f_{Q S S} d \theta d p & =e+\frac{M_{Q S S}^{2}-1}{2}, \\
\int_{-\pi}^{\pi} \int_{-\infty}^{\infty} \eta(\theta, p)^{n} f_{Q S S} d \theta d p & =\epsilon_{n} \quad \forall n \geqslant 2, \\
\int_{-\pi}^{\pi} \int_{-\infty}^{\infty} \cos (\theta) f_{Q S S} d \theta d p & =\left(M_{Q S S}\right)_{x}, \\
\int_{-\pi}^{\pi} \int_{-\infty}^{\infty} \sin (\theta) f_{Q S S} d \theta d p & =\left(M_{Q S S}\right)_{y},
\end{aligned}
$$

where we recall that $f_{Q S S}$ depends self-consistently on $\left(\beta, \mu, v_{n},\left(M_{Q S S}\right)_{x},\left(M_{Q S S}\right)_{y}\right)$, as dictated by relation (12). In
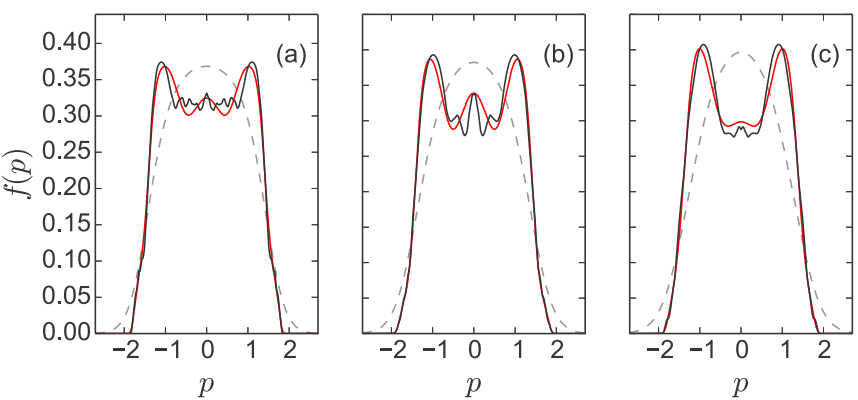

FIG. 2. The velocity distribution profiles are displayed for $e=$ 0.9 and different choices of the initial magnetization (from left to right, $\left.M_{0}=0.3,0.5,0.7\right)$. In all cases the system is predicted to converge to a homogeneous QSS. The result of the Vlasov simulations is depicted by a black solid line. It is averaged over the latter half of the simulation time. The standard Lynden-Bell theory yields the gray dashed curve. The modified maximum entropy scheme with the inclusion of the additional pseudoconserved quantities yields the profiles represented by red solid lines.

agreement with the original Lynden-Bell scheme, the generalized conditions (13) predict a parameter space $\left(M_{0}, e\right)$ partitioned into two regions, corresponding to magnetized and homogeneous QSSs. Above the threshold, when the QSS magnetization is about zero, Eq. (12) reduces to the following compact expression, which is only function of $p$ (and the so-called velocity distribution):

$$
f_{Q S S}(p)=f_{0} \frac{e^{-\beta p^{2} / 2-\sum_{n} v_{n}\left(p^{2} / 2\right)^{n}-\mu}}{1+e^{-\beta p^{2} / 2-\sum_{n} v_{n}\left(p^{2} / 2\right)^{n}-\mu}},
$$

which can be interpreted as an asymptotic free theory. $f_{Q S S}(p)$ is thus defined by $f_{0}, \beta, \mu$, and $v_{n}$ for $n \geqslant 2$. The force field acting during the violent relaxation phase is implicated in the short-time modulation of $\left\langle\eta(\theta, p ; t)^{n}\right\rangle$ and indirectly enters the picture through the quantities $\epsilon_{n}$. In this respect, the proposed approach to the QSS resembles a quantum quench from interacting to free theory, as for instance discussed in Ref. [26], or, more recently, in Ref. [27] where the inspected system is shown to equilibrate to a Generalised Gibbs Ensemble [28]. In Fig. 2 the velocity distribution $f(p)=\int f(\theta, p) d \theta$ as recorded from Vlasov simulations is plotted (black solid line) in the region of a homogeneous QSS for different values of the initial magnetization. The obtained profiles display the two characteristic bumps to which we alluded above. Other collective structures are, however, present and become more evident when $M_{0}$ is made larger for a fixed energy amount [Fig. 2(c)]. To favor the comparison between theory and simulations, we follow exactly the same strategy as outlined in [16]: the numerical distribution is averaged over a finite time of observation.

The velocity distribution predicted by the standard LyndenBell theory (dashed gray line) fails to capture the fine details of the simulated profile. In contrast, the generalized theory here described [see Eq. (12)], which sets the even average momenta to the asymptotic values determined by Vlasov dynamics, proves definitely more adequate in explaining the observations (solid red line). The macroscopic details of the velocity profile are adequately captured upon truncation at the tenth order in the hierarchy of pseudoconserved momenta. Notice that 

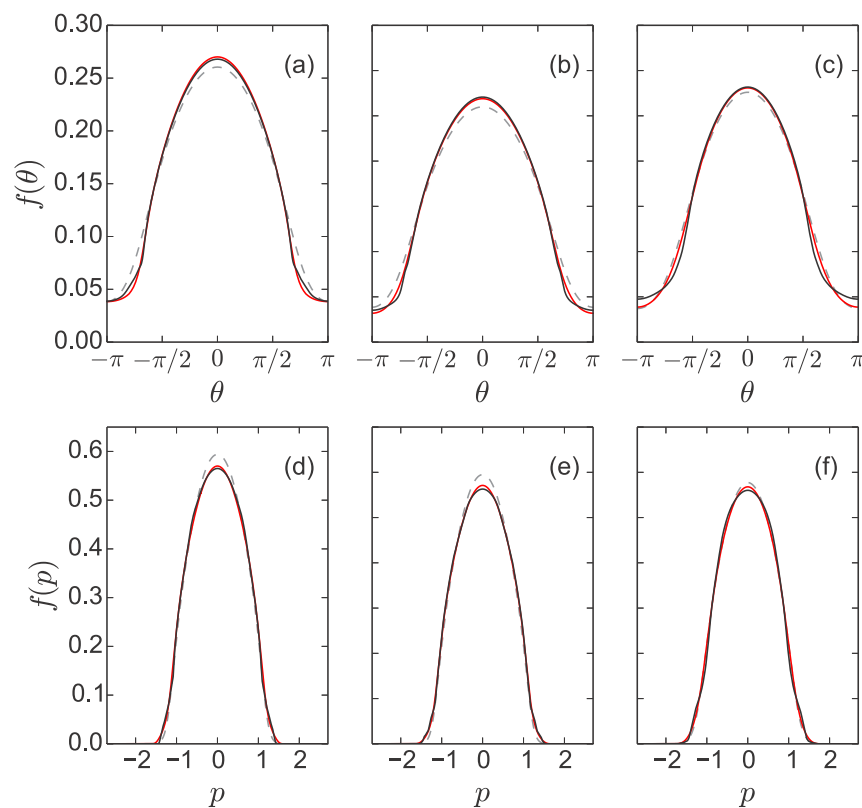

FIG. 3. The velocity distribution profiles are plotted for $e=0.6$ and different choices of the initial magnetization (from left to right, $\left.M_{0}=0.3,0.5,0.7\right)$. The system converges to a magnetized QSS. The result of the Vlasov simulations are drawn with a black solid line (averaged, as in Fig. 2). The standard Lynden-Bell theory yields the gray dashed curve, whose deviations from the simulation results are clearly visible. Predictions based on the modified maximum entropy scheme with the inclusion of the additional pseudoconserved quantities are depicted by red solid lines. The latter are almost identical to the simulated profiles for $M_{0}=0.3$ and 0.5 and still improve on the standard Lynden-Bell result for $M_{0}=0.7$.

the improvements are evident not only in the bulk of the distribution, where the resonant particles are trapped, but also in the tails, with the theoretical curves adhering better to the corresponding numerically computed lines. Smaller coherent structures appear in the numerics which are not captured by the theory at this level of resolution. Accounting for higher moments or additional pseudoconserved charges might help to ameliorate the agreement even further. The inclusion of additional dynamical constraints is also beneficial in the region of magnetized QSSs (despite the approximations involved in the variational analysis), as can be appreciated by visual inspection of Fig. 3.

\section{CONCLUSIONS}

In conclusion we have here discussed a generalization of the maximum entropy principle due to Lynden-Bell and focused on explaining the emergence of quasistationary states in systems subject to long-range forces. Imposing additional dynamical constraints, stemming from the Vlasov equation that rules the evolution of the system in the continuum limit, allowed us to considerably enhance the predictive ability of the theory. In particular, by constraining the asymptotic values of the even moments of the velocity distribution, we theoretically demonstrated that the existence of two (or multiple) islands in the single-particle phase space is compatible with a maximum entropy principle. Our conclusion is twofold. On the one side, we confirmed that a statistical mechanics treatment based on the governing Vlasov equation can be successfully invoked to address QSS peculiarities. Discrepancies between empirical observation and the standard Lynden-Bell theory can be significantly reduced by taking explicit note of key dynamical restrictions. On the other side, we expect that our results could open up novel avenues to investigate those systems of both theoretical and experimental relevance where long-range forces are active and QSSs have been observed (e.g., plasmas, Coulomb systems). Further work is required to obtain a consistent estimate of the additional constraints imposed, therefore yielding a fully predictive theoretical scenario.

\section{ACKNOWLEDGMENTS}

D.F. acknowledges financial support from the program PRIN 2012 financed by the Italian MIUR. Gabriele Martelloni acknowledges the ERC for financial support under Starting Grant 279391 EDEQS. P.d.B. acknowledges support from a Postdoctoral Fellowship of the Research Foundation-Flanders (FWO). The authors thanks P. Di Cintio and S. Ruffo for stimulating discussions.
[1] A. Campa, T. Dauxois, D. Fanelli, and S. Ruffo, Physics of Long Range Interacting Systems (Oxford University Press, Oxford, 2015).

[2] A. Campa, T. Dauxois, and S. Ruffo, Phys. Rep. 480, 57 (2009).

[3] D. R. Nicholson, Introduction to Plasma Physics (Krieger, Malabar, FL, 1992).

[4] T. Padmanabhan, Phys. Rep. 188, 285 (1990).

[5] J. Barré, T. Dauxois, G. De Ninno, D. Fanelli, and S. Ruffo, Phys. Rev. E 69, 045501(R) (2004).

[6] T. N. Teles, D. Fanelli, and S. Ruffo, Phys. Rev. E 89, 050101(R) (2014).

[7] D. Lynden-Bell, Mon. Not. R. Astr. Soc. 136, 101 (1967).

[8] D. Lynden-Bell and R. Wood, Mon. Not. R. Astron. Soc. 138, 495 (1968).

[9] A. Antoniazzi, D. Fanelli, J. Barré, P. H. Chavanis, T. Dauxois, and S. Ruffo, Phys. Rev. E 75, 011112 (2007).
[10] A. Antoniazzi, D. Fanelli, S. Ruffo, and Y. Y. Yamaguchi, Phys. Rev. Lett. 99, 040601 (2007).

[11] F. Staniscia, P. H. Chavanis, G. De Ninno, and D. Fanelli, Phys. Rev. E 80, 021138 (2009).

[12] R. Pakter and Y. Levin, Phys. Rev. Lett. 106, 200603 (2011).

[13] I. B. Bernstein, J. M. Greene, and M. D. Kruskal, Phys. Rev. 108, 546 (1957).

[14] P. de Buyl, D. Mukamel, and S. Ruffo, Phys. Rev. E 84, 061151 (2011).

[15] M. Antoni and S. Ruffo, Phys. Rev. E 52, 2361 (1995).

[16] A. Antoniazzi, F. Califano, D. Fanelli, and S. Ruffo, Phys. Rev. Lett. 98, 150602 (2007).

[17] R. Bachelard, C. Chandre, D. Fanelli, X. Leoncini, and S. Ruffo, Phys. Rev. Lett. 101, 260603 (2008).

[18] Y. Y. Yamaguchi, Phys. Rev. E 84, 016211 (2011). 
[19] Y. Y. Yamaguchi, J. Barré, F. Bouchet, T. Dauxois, and S. Ruffo, Phys. A (Amsterdam, Neth.) 337, 36 (2004).

[20] P. H. Chavanis, Phys. A (Amsterdam, Neth.) 359, 177 (2006).

[21] J. Michel and R. Robert, Commun. Math. Phys. 159, 195 (1994).

[22] P. de Buyl, Comput. Phys. Commun. 185, 1822 (2014); VMF90, https://github.com/pdebuyl/vmf90.

[23] The hypothetical possibility of obtaining exotic features of the velocity distribution function from the inclusion of additional exactly conserved invariants has also been considered in an abstract setting in [29].
[24] M. Perin, C. Chandre, P. J. Morrison, and E. Tassi, Phys. Plasmas 22, 092309 (2015).

[25] The obtained distribution function depends only on the oneparticle energy $\eta(\theta, p)$. Hence it is by definition stationary under the Vlasov dynamics and also in the magnetized regime [14].

[26] S. Sotiriadis and P. Calabrese, J. Stat. Mech. (2014) P07024.

[27] S. Sotiriadis and G. Martelloni, J. Phys. A: Math. Theor. 49, 095002 (2016).

[28] M. Rigol, V. Dunjko, V. Yurovsky, and M. Olshanii, Phys. Rev. Lett. 98, 050405 (2007).

[29] P. de Buyl, D. Mukamel, and S. Ruffo, Philos. Trans. R. Soc. A 369, 439 (2011). 